\title{
Whole-Genome Sequencing Detection of Ongoing Listeria Contamination at a Restaurant, Rhode Island, USA, 2014
}

\section{Jonathan S. Barkley, Michael Gosciminski, Adam Miller $^{1}$}

In November 2014, the Rhode Island Department of Health investigated a cluster of 3 listeriosis cases. Using wholegenome sequencing to support epidemiologic, laboratory, and environmental investigations, the department identified 1 restaurant as the likely source of the outbreak and also linked the establishment to a listeriosis case that occurred in 2013.

$I^{1}$ nfection with Listeria monocytogenes, a foodborne bacterial pathogen, causes listeriosis, which can lead to severe illness, typically among persons with compromised immune systems and pregnant women and their fetuses. The pathogen can survive at high salt concentrations and grow at refrigeration temperatures (1). These properties enable the bacteria to persist in food processing and food service establishments for extended periods. Listeriosis has a long incubation period (3-70 days), making exposure recall difficult. Retail delicatessens are a potential source of L. monocytogenes because they hold ready-to-eat foods at refrigeration temperatures; however, a risk assessment by the United States Department of Agriculture's Food Safety and Inspection Service suggests that thorough sanitization of food contact surfaces, proper maintenance of equipment and facilities, safe product handling practices, and good employee practices to avoid cross-contamination can help prevent listeriosis cases associated with retail food establishments (2).

Since 1998, PulseNet (http://www.cdc.gov/pulsenet/ index.html) has used pulsed-field gel electrophoresis (PFGE) to look at genetic differences in L. monocytogenes subtypes and to identify outbreaks. However, distantly related strains can appear indistinguishable by PFGE; thus, greater differentiation may be needed to distinguish between outbreak and sporadic cases of listeriosis. Wholegenome sequencing (WGS) offers an opportunity to further discriminate between strains and identify outbreaks. WGS has historically been used retrospectively to provide additional insight into outbreak investigations (3). However, since September 2013, WGS has been performed

Author affiliation: Rhode Island Department of Health, Providence, Rhode Island, USA

DOI: http://dx.doi.org/10.3201/eid2208.151917 on all clinical L. monocytogenes isolates identified in the United States by the Centers for Disease Control and Prevention (Atlanta, GA) and several state public health laboratories (4). L. monocytogenes is a good candidate for WGS because it causes a relatively rare condition that can result in serious illness, it has a small genome that is relatively easy to analyze, and epidemiologic surveillance and food regulatory program components for the bacterium are strong (5).

Data obtained from WGS has been analyzed using whole-genome multilocus sequence typing (wgMLST), a technique that examines allelic differences from thousands of loci, and $\approx 96 \%$ of $L$. monocytogenes coding sequences have been identified as loci in the wgMLST scheme (S. Stroika, Centers for Disease Control and Prevention, pers. comm., 2016 Jan 29). To discriminate between strains and identify outbreaks, alleles within the coding sequence (i.e., loci) are compared with $\approx 178$ reference genomes. A unique combination of alleles at each locus specifies the sequence type, which enables comparison of isolates (6); the smaller the number of allelic differences between isolates, the more related they are.

The Rhode Island Department of Health (RIDOH) attempts interviews and, when applicable, conducts environmental investigations for all reports of listeriosis. Each year during 2011-2013, RIDOH received $\approx 3$ reports of listeriosis, most of which were sporadic cases. However, in November 2014, a cluster of cases was detected from laboratory reports and examined using WGS in conjunction with epidemiologic, laboratory, and environmental investigations. Isolates were confirmed to be L. monocytogenes and submitted for PFGE analysis. The Centers for Disease Control and Prevention performed WGS on clinical isolates; the Food and Drug Administration performed WGS on food isolates.

\section{The Investigation}

During October 27-November 5, 2014, RIDOH's Center for Acute Infectious Disease Epidemiology was notified of $3 \mathrm{~L}$. monocytogenes-infected persons residing in the same city. The 3 case-patients were all non-Hispanic white persons $>60$ years of age; 2 had an immunocompromising condition. Interviews conducted by the Center for Acute Infectious Disease Epidemiology identified a single

${ }^{1}$ All authors contributed equally to this article. 
common restaurant visited by the 3 patients. RIDOH's Center for Food Protection performed inspections and collected food and environmental samples at the establishment.

PFGE analysis showed that clinical L. monocytogenes isolates from the 3 case-patients shared an identical, common PFGE pattern (Figure). To determine the relationship between the isolates, RIDOH collaborated with federal partners to conduct WGS. Results of wgMLST showed that the isolates were closely related $(0-5$ allelic differences) (Figure) and a close genetic match (median allelic differences 4) to a clinical isolate from a 2013 patient, who was reinterviewed and reported eating at the same restaurant. A sliced prosciutto sample from the restaurant tested positive for L. monocytogenes, and PFGE patterns for this isolate matched those for isolates from the 2013 and 2014 case-patients. Results of wgMLST showed that the isolate from the prosciutto differed by $0-5$ alleles (median 3 ) from the 2014 clinical samples and by $0-11$ alleles (median 4) from the 2013 clinical sample (Figure). Sequences for the isolates were uploaded to GenBank (7) (clinical isolates: accession nos. SAMN02400177, SAMN03253348-49, SAMN03253359; isolate from prosciutto: accession no. SAMN03218571).

A total of 10 food and environmental food samples were initially collected from the restaurant. Swab samples were obtained from the food slicer, preparation tables, and walk-in cooler. Environmental investigation of the restaurant identified issues related to control of L. monocytogenes: the temperature of the refrigerated unit that held sliced meat and other food items was elevated $\left(52^{\circ} \mathrm{F}\left[11^{\circ} \mathrm{C}\right]\right)$, and cleanliness issues were observed with the preparation tables and slicer. An additional 19 environmental samples were later collected from the establishment; however, the refrigerated unit and preparation tables had been replaced, so additional swab samples could not be collected from those surfaces. The sample of sliced prosciutto was the only L. monocytogenes-positive sample identified at the restaurant; however, just 1 of the 2014 case-patients reported eating prosciutto (in an antipasto salad) at the restaurant. Other foods reported included green salad and coleslaw.
RIDOH tested a sample of prosciutto from an unopened package from the establishment and collaborated with the Food Safety and Inspection Service to see if the processing plant had recently tested positive for L. monocytogenes. The sample tested negative, and no positive tests had been reported at the plant in at least 1 year.

\section{Conclusions}

Epidemiologic, environmental, and laboratory investigation results implicated a restaurant with sanitation issues and improper sliced meat storage as the likely source of a multiyear listeriosis outbreak. A long incubation period makes WGS an effective technology to use during listeriosis outbreak investigations and to identify outbreakassociated cases originally believed to be sporadic cases. This technology can help overcome difficulties associated with investigating listeriosis cases and can be useful for the investigation of other pathogens. In this investigation, WGS (wgMLST) helped link the 2013 listeriosis case, which was originally believed to be a sporadic case, to the 2014 outbreak. Furthermore, given that the 4 isolates had a common PFGE pattern, this technology increased confidence that the restaurant, which was the only common restaurant among the 4 patients, was the source of the outbreak. The allelic differences observed are consistent with slow, spontaneous mutation occurring over a long period due to persistent contamination.

There is no set number of allelic differences used to determine whether clusters of cases are part of actual outbreaks (8). Thus, WGS is not sufficient by itself to identify outbreaks and must be performed in conjunction with epidemiologic, laboratory, and environmental investigations $(8,9)$. In the investigation we describe, WGS was used in this supporting role. The close relationship that WGS showed between the clinical isolates and the isolate from meat provides additional evidence that the restaurant was the likely source of contamination for the cases in 2013 and 2014.

Our findings support the need to control L. monocytogenes at retail food establishments. Storing meat at $\leq 41^{\circ} \mathrm{F}$

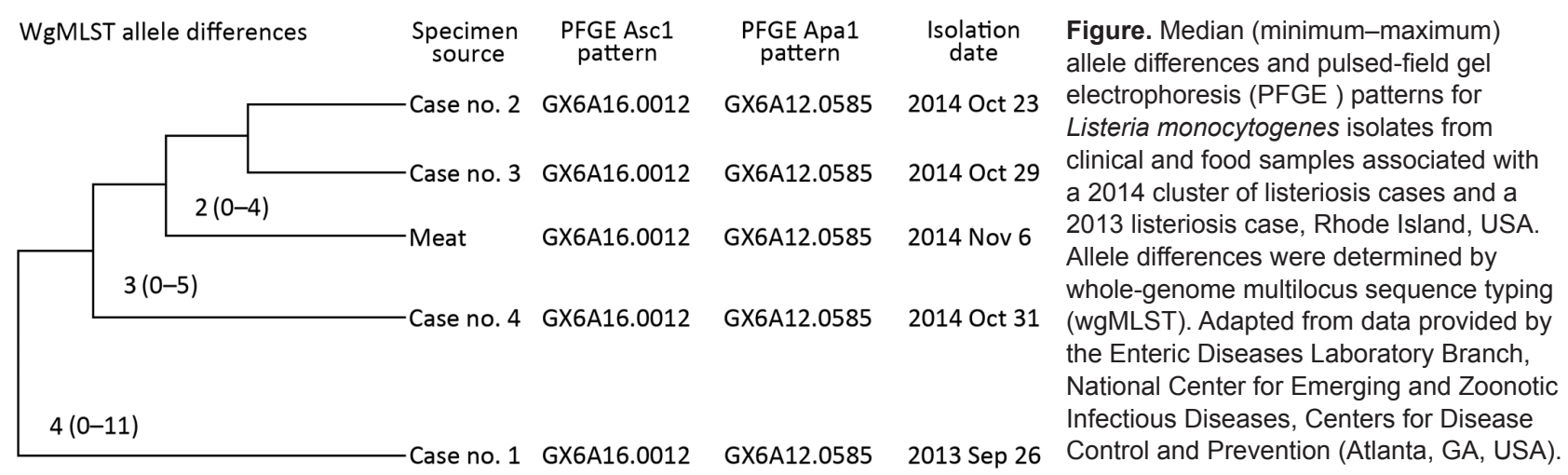


$\left(5^{\circ} \mathrm{C}\right)$ can prevent $\approx 9 \%$ of listeriosis cases (2). In addition, retail delicatessens and food establishments can prevent $L$. monocytogenes-associated illnesses among customers by controlling cross-contamination, cleaning and sanitizing food contact surfaces, and eliminating environmental niches.

\section{Acknowledgments}

We thank the Rhode Island State Laboratory for performing confirmatory L. monocytogenes testing on clinical and food samples and for coordinating PFGE and WGS testing; the Massachusetts William A. Hinton State Laboratory for performing PFGE testing of the clinical and food samples; and the Centers for Disease Control and Prevention and Food and Drug Administration for performing WGS of clinical and food samples, respectively.

Mr. Barkley is a public health epidemiologist at the Center for Food Protection, Rhode Island Department of Health. His research interests include understanding risk factors of foodborne illness associated with retail food establishments.

\section{References}

1. Ferreira V, Wiedmann M, Teixeira P, Stasiewicz MJ. Listeria monocytogenes persistence in food-associated environments: epidemiology, strain characteristics, and implications for public health. J Food Prot. 2014;77:150-70. http://dx.doi.org/10.4315/0362-028X.JFP-13-150

2. United States Department of Agriculture, Food Safety and Inspection Service. Best practices guidance for controlling Listeria monocytogenes in retail delicatessens. June 2015 [cited 2016 Feb 1]. http://www.fsis.usda.gov/wps/wcm/connect/29d512580651-469b-99b8-e986baee8a54/Controlling-LM-Delicatessens. pdf?MOD=AJPERES

3. Le VT, Diep BA. Selected insights from application of wholegenome sequencing for outbreak investigations. Curr Opin Crit Care. 2013;19:432-9. http://dx.doi.org/10.1097/MCC.0b013e3283636b8c

4. Centers for Disease Control and Prevention. Advanced molecular detection (AMD). AMD projects: learning from Listeria [cited 2015 Oct 23]. http://www.cdc.gov/amd/project-summaries/ listeria.html

5. Jackson B, Jackson K, Tarr C, Evans P, Klimke W, Kubota K, et al. Improving detection and investigation of listeriosis outbreaks using real-time whole-genome sequencing. Presented at: IDWeek 2014; Philadelphia, PA, USA; 2014 Oct 8-12.

6. Larsen MV, Cosentino S, Rasmussen S, Friis C, Hasman H, Marvig RL, et al. Multilocus sequence typing of total-genomesequenced bacteria. J Clin Microbiol. 2012;50:1355-61. http://dx.doi.org/10.1128/JCM.06094-11

7. Benson DA, Clark K, Karsch-Mizrachi I, Lipman DJ, Ostell J, Sayers EW. GenBank. Nucleic Acids Res. 2015;43:D30-5. http://dx.doi.org/10.1093/nar/gku1216

8. Jackson B. Everything in sequence: listeriosis outbreak investigations in the era of WGS. Presented at: Integrated Foodborne Outbreak Response and Management (InFORM) 2015 Conference; Phoenix, AZ, USA; 2015 Nov 17-20.

9. Leekitcharoenphon P, Nielsen EM, Kaas RS, Lund O, Aarestrup FM. Evaluation of whole genome sequencing for outbreak detection of Salmonella enterica. PLoS ONE. 2014;9:e87991. http://dx.doi.org/10.1371/journal.pone.0087991

Address for correspondence: Jonathan S. Barkley, Rhode Island Department of Health, Center for Food Protection, 3 Capitol Hill, Rm 203, Providence, RI 02908-5097, USA; email: jonathan.barkley@health.ri.gov

\section{April 2015: Emerging Viruses Including:}

- Reappearance of Chikungunya, Formerly Called Dengue, in the Americas

- Hantavirus Pulmonary Syndrome, Southern Chile, 1995-2012

- Animal-Associated Exposure to Rabies Virus among Travelers, 1997-2012

- Evolution of Ebola Virus Disease from Exotic Infection to Global Health Priority, Liberia, Mid-2014

- Population Structure and Antimicrobial Resistance of Invasive Serotype IV Group B Streptococcus, Toronto, Ontario, Canada

- Norovirus Genotype Profiles Associated with Foodborne Transmission, 1999-2012

- Sequence Variability and Geographic Distribution of Lassa Virus, Sierra Leone

- Deaths Associated with Respiratory Syncytial and Influenza Viruses among Persons $\geq 5$ Years of Age in HIV-Prevalent Area, South Africa, 1998-2009

- Highly Pathogenic Avian Influenza A(H5N1) Virus Infection among Workers at Live Bird Markets, Bangladesh, 2009-2010

- Increased Risk for Group B Streptococcus Sepsis in Young Infants Exposed to HIV, Soweto, South Africa, 2004-2008

- La Crosse Virus in Aedes japonicus japonicus Mosquitoes in the Appalachian Region, United States

- Pathogenicity of 2 Porcine Deltacoronavirus Strains in Gnotobiotic Pigs

- Multidrug-Resistant Salmonella enterica Serotype Typhi, Gulf of Guinea Region, Africa

\section{http://wwwnc.cdc.gov/eid/articles/issue/21/4/table-of-contents}

